\title{
Effects of Different Lighting Arrangements on Space Perception
}

\author{
Banu Manav", Cengiz Yener ${ }^{*+}$
}

The idea to improve the quality of lighting design in interior spaces bas gained importance in the last years. This experimental study concentrates on the quality of lighting and the relation between lighting arrangements and perception. A room baving four different ligbting arrangements was prepared and the differences betuceen the perception of the participants under each ligbting arrangement was analysed.

The results suggested that different lighting arrangements affect perception. Wall washing enbanced the impressions of clarity and order, cove lighting enbanced spaciousness and order and uplighting made the same space relaving. private and pleasant.

\section{Introduction}

It would be impossible to perceive our surroundings in the absence of light. Lighting- either natural or artificial-plays an important role in our lives. Such an important property can enhance or subdue the architectural features, and is therefore, be considered and planned as a part of the architecture.

When an observer looks at a lighting system, a reaction arises that can be aestherical or emotional [1] . The aesthetical response requires attention to the entire area and is an expression of immediate reaction such as good or bad, too bright or too dark [2] . The emotional reaction, on the other hand, is an expression of a feeling that occurs after being present in the environment for some time. Emotional reactions are related to the combination of many senses such as pleasantness, relaxation, depression, etc.

These reactions are subjective agreements based on certain situations, so it is not possible to reach objective solutions. Everyone has a different physical condition, cultural background, demands and beliefs which in turn affect emotional or aesthetical responses to lighting systems. Emotional and aesthetical impressions are accomplished with the color. materials, textures in a space as well, but the usage of the right light in the right place is very important.

'Frauly of Art, Design \& Architecture, Bilkent Liniversity. 06533 Bilkent. Ankara, Turker " Assoc. Prof. Faculy of Art, Design \& Architecture, Bilkent University. Ankara. Turker 'Comesponding author.

\section{The Aim}

This study tries to find out how effective the lighting strstems are on the perception of the users. Six impressions, namely clarity. spaciousness (roominess), relaxation, privacy (intimacy), pleasantness and order were tested. As perception is a subjective interpretation. the relationship between these impressions and other factors such as age. sex differentials and eve defects are searched for in the same setting under different lighting arrangements.

\section{Previous Studies}

As the interest in environmental psychokggy increased in 1970 0 , different variables related to light gained importance. One of these was the quality of lighting in the built entironment. Quality of lighting was relared to the quality of life that aimed to eliminate the factors causing stress such as , Iare, distortion and the like. It tried to impirtse user"s well being. motivation and performance.

A pioneer in the field was John Flynn. who passed away in 1980. A series of studies conducted bi Flinn and his colleagues in 19:3 [3] suggest that lighting conditions can affect moxds. A medium-sized conference rox)m that had various lighting s!stems was prepared. Rating scale judgements were obtained under each lighting arrangenent and the results showed a significant difference under different lighting strstens. Five factors tested for the study were, evaluative impressions, perceptual clarity spatial complexiry (visual noise). spaciousness and formality (style. fashion). 
ments [4]. Subjects evaluated the slides without observing the real spaces. The results showed that the reliable judgments of a space were not stimulus bound by that space, because the results were identical.

Another study by Flynn in 1974 [5] also showed that the same lighting systems can give rise to the same impressions in different settings. For the study, three rooms were arranged, each having the same lighting systems and controlled by multiple switches, so that intensities, color tempera. ture and distribution of light were consistent among the rooms. One room was medium-sized and was irregular in shape, the second one was large and rectangular and the third one was medium-sized and rectangular. The lighting modes were as follows; a) overhead fluorescent, b) only four walls were illuminated, c) overhead fluorescent and four walls were illuminated, d) overhead incandescent downlights, e) overhead incandescent downlights and only one wall was illuminated. The impressions of perceptual clarity, spaciousness, relaxation, privacy, pleasantness and complexity were evaluated. It was concluded that, there were not significant inconsistencies except for the impressions of relaxation and privacy. This inconsistency could be related to the nonuniform distribution of light pattems. As one of the rooms was irregular in shape, nonuniform lighting did not affect the side walls, those parts were darker and this increased the feeling of unpleasantness and tension.

As cited in Butler and Biner [6], in 1979, Danford et al. criticised Flynn's research, they emphasized that it had narrow focus and factors like satisfaction, perceived well-being were difficult to quantify and integrate with the illumination level. However, Flynn's findings are accepted by many researchers.

Smith [7], Baron and Rea [8] and Loe [9] who had studies that evaluate the relation between the lighting arrangements and user performance shared the same views with Flynn. Baron and Rea concluded that people reported more positive feelings under low illuminance levels and warm light. The results of Smith's research suggested that as illumination level increased, the space looked more spacious.

In Loe's study [9] , twelve observers were asked to make subjective assessments of an interior, lighted in eighteen different ways. The experiment showed that people prefer interiors that have higher illumination levels. The light pattern was also accepted to be an important aspect of a successful design.

Another study about the impressions is by Hughes and his associates [10] in which the concept of visual claricy under different light sources of various colour temperatures was examined. According to the results, higher color temperature lamps were preferred from visual clarity point of view.

Aksugür [11] investigated how spaciousness changes in relation to the characteristics of the selected lamps. Two different light sources (rungsten-filament lamps and daylight fluorescent tubes) were selected and their effect on the impression of spaciousness was analysed. It was claimed that, spaciousness increases under daylight fluorescent tubes rather than tungsten-filament lamps.

\section{Experimental Set-Up}

The present study was carried out at Bilkent University, Deparment of Interior Architecture and Environmental Design, at Ankara, Turkey. The size of room in which the experiments are carried out was $4.10 \times 4.18 \mathrm{~m}$, approximately 17 square meters; ceiling height is $3.84 \mathrm{~m}$. The floor was covered by $30 \times 30 \mathrm{~cm}$ terrazzo tiles, walls and ceiling were painted white.
There were no windows. This room was chosen mainly for two reasons. The first reason was, since there were no windows, there was no daylight penetration therefore the change in the atmosphere due to artificial lighting arrangements could be evaluated easily. The second reason was, the room was not too big and this provided easier and less costly lighring installations.

The original lighting in the room was provided by two $40 \mathrm{~W}$ (Philips TI54) fluorescent lamps which do not have reflectors and were installed on the ceiling. The lighting system and the arrangement of the room were changed for the purposes of the study. In addition to the existing fluorescent lamps, cove lighting, wall washing and uplighting systems were installed. Then, the four lighting systems were connected into a switch box and were operated from the observation table.

The wall washing and cove lighting were installed by 40W and 20W (Philips TL54) fluorescent lamps that had a color temperature (CT) of $6200 \mathrm{~K}$ and a color rendering index (CRT) of 72 . Fluorescent lamps were installed on wall brackets which were $60 \mathrm{~cm}$ below the ceiling. The inside of these brackets have been painted white in order to increase the reflectivity and to get more light output.

Uplighting was achieved by two torcheres having $300 \mathrm{~W}$ tungsten halogenated lamps each. The color temperature of tungsten halogenated lamps was about $3000 \mathrm{~K}$ and color rendering was accepted as 100 . Torcheres were located behind the observation table, directed towards the ceiling and walls that result in non-uniform space lighting. After the lighting systems were installed, the room was arranged as a study room. The redesigned state of room can be seen in Fig. 1 .

\section{Subjects}

174 undergraduate universiry students, 85 males and 89 females ranging in age from 18-27 years participated in this study. They were all from freshman and sophomore classes at the Bilkent University, Department
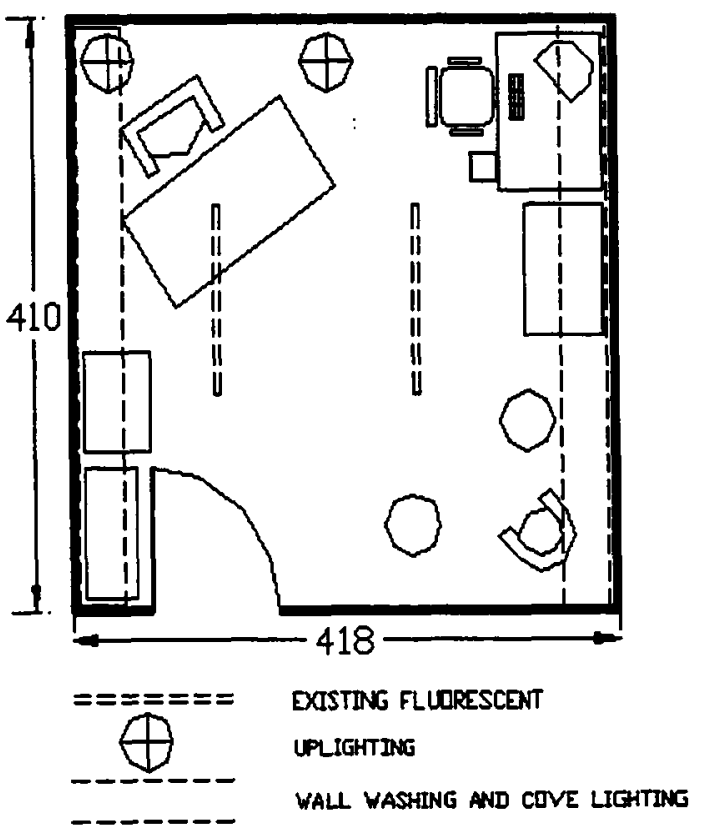

Figure 1. The plan of the room 


\section{Subjects}

174 undergraduate universitystudents, 85 males and 89 females ranging in age from 18-27 years participated in this study. They were all from freshman and sophomore classes at the Bilkent University, Department of Interior Architecture and Environmental Design and had not taken any courses on lighting at the time of the study. No personal data (except their age, gender and the type of eye defects) was collected. Each experimental session lasted for about 6 minutes.

\section{The Procedure}

Standardisation of procedure was achieved by the following steps;

- The room was not illuminated when the participants entered.

- They entered the room one by one.

- They were allowed to tum on/off the lighting systems as much as they desired, but not allowed to turn on more than one lighting system at a time.

- They filled in the questionnaires at the observation table.

The questionnaire consisted of nine questions about the impressions of clarity, spaciousness, relaxation, privacy, pleasantness and order was given to each participant and they were asked to determine the most suitable lighting system for each impression. Numerical and graphical results of the quetionnaires are shown on Figure 2 and on Table 1.

\section{Experimental Results}

\section{The impression of clarity}

Clarity is a subjective feeling that is related to the appearance of objects. This can differ due to the lighting distribution in the room and the distance of the objects from the observer. With the first two questions, the evaluation of the appearance of the objects were asked. Wall washing has been found the most preferred one out of the four lighting ststems in the room.

\section{The impression of spaciousmess}

The second impression is spaciousness (roxminess) that is related to the personal judgments about the dimensions of a space. The third and the fourth questions were abour the lighting sistems that make the room appear larger and the ceiling higher respectively. Cove lighting has heen found as the most suitable lighting st!stem to enhance the feeling of spaciousness.

\section{The impression of relaxation}

The fifth question was related to the impression of relaxation. Subjects were asked to select the most suitable lighting system that arouse the feeling of relaxation. According to the responses of the participants. tungsten-halogenated lamps were found to make the space norte relaxing among the others.

\section{The impression of pritacy}

The sixth question was about the impression of priacy. a condition that arises the feeling of belonging to a single individual. Tungsten-halogenated lamps were evaluated as the most suitable lighting system for enhancing the feeling of privacy.

\section{The impression of pleascintmess}

With the seventh question. the lighting șstem that makes the r(x)m pleasant was asked. According to responses. tungsten-halogenated torcheres were the ones that made the room most pleasing.

\section{The impression of order}

The last no questions were about order that is related to the compatibiliny of the lighting system with the functions in the room. The most suitable lighting system for a study room was asked with question eight. Out of these four lighting sustems, cove lighting has been found the most

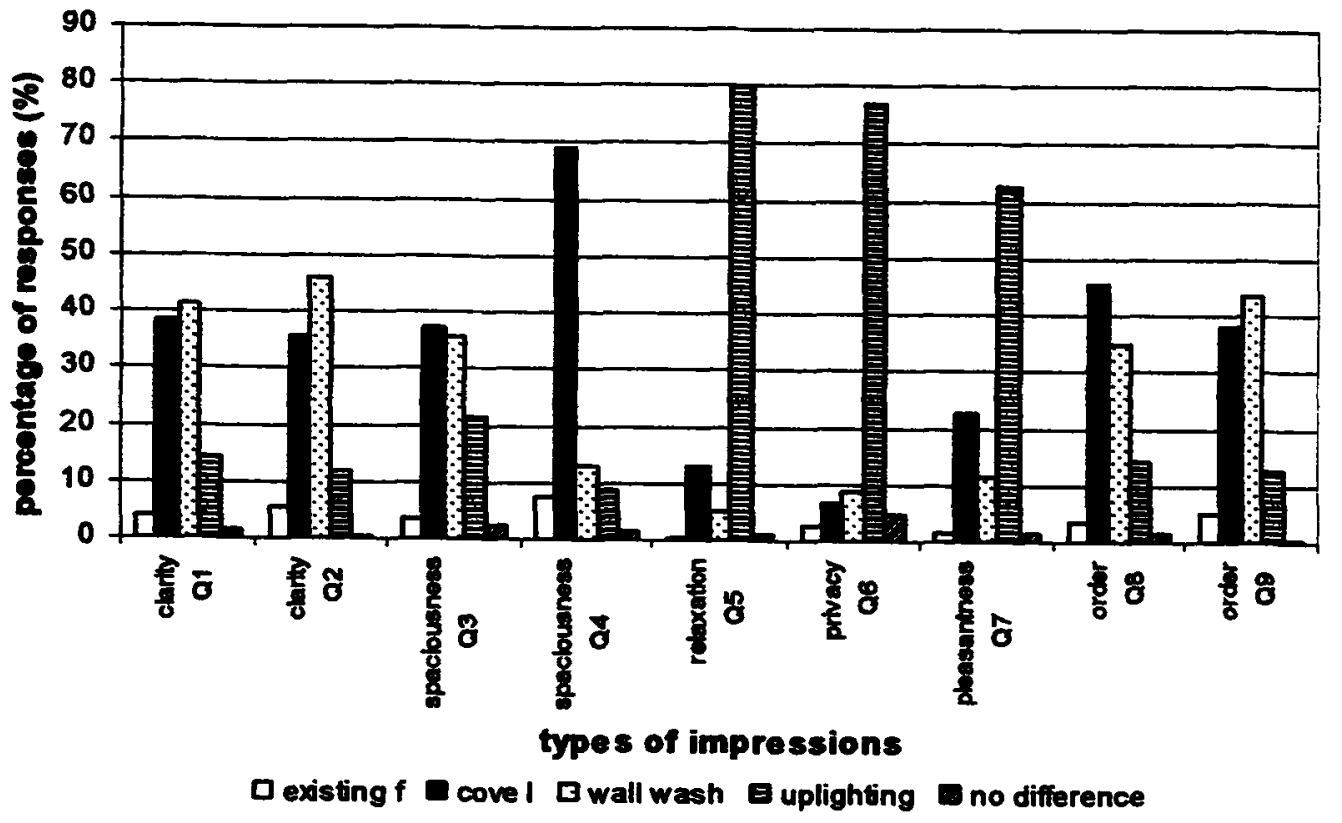

Figure 2. Comparision of the responses for each lighting arrangement 
Table1

Number of responses for Each Question

\begin{tabular}{|lllllll|}
\hline & 1 & 2 & 3 & 4 & no difference \\
\hline $\begin{array}{l}\text { Q1. Under which lighting, is the appearance of } \\
\text { the objects on the table most clear? }\end{array}$ & 7 & 67 & 72 & 25 & 3 \\
$\begin{array}{l}\text { Q2. Under which lighting, is the appearance } \\
\text { of the objects in the room most clear? }\end{array}$ & 10 & 62 & 80 & 21 & 1 \\
$\begin{array}{l}\text { Q3. Under which lighting, the room appears } \\
\text { larger? }\end{array}$ & 6 & 65 & 62 & 37 & 4 \\
$\begin{array}{l}\text { Q4. Under which lighting, the ceiling appears } \\
\text { higher? }\end{array}$ & 13 & 120 & 23 & 15 & 3 \\
$\begin{array}{l}\text { Q5. Under which lighting, is the room most } \\
\text { relaxing? }\end{array}$ & 1 & 23 & 9 & 139 & 2 \\
$\begin{array}{l}\text { Q6. Under which lighting, the room appears } \\
\text { more private? }\end{array}$ & 5 & 12 & 15 & 134 & 8 \\
Q7. Which of these lighting systems is the \\
most pleasing one?
\end{tabular}

suitable one for a study room. Finally, with the last question the participants were asked to choose the lighting sustem that has been designed according to the function in the room and wall washing was selected.

\section{Statistical Analysis}

The results were analysed by Chi-square test and One-way ANOVA.

The evaluation of the differences between the mean values of the responses for the ligbting systems and the impressions

The mean scores for the lighting arrangements (existing fluorescent tubes, cove lighting, wall washing, uplighting, no difference) of room are; $6.66,59.22,58.55,3.12$ and total sample mean is 34.8. Computed $F$ value (5.4519) is bigger than $\mathrm{F}$ table value $(2.61), \mathrm{H}_{11}$ is rejected. Therefore, it can be said that, there are differences between the mean values of the responses for the lighting arrangements. Each lighting system arises different impression which in turm affects perception.

\section{The evaluation of the relation between the age of the participants and the lighting systems}

Chi-square distribution is 30.57574 that is bigger than the computed $c^{2}$ ( $d f=8, a=0.050)=15.51$, therefore the null hypothesis is rejected at the 5 percent level of significance, there is a relationship between the age of the participants and the lighting systems. Perception of a space is not independent of age differentials. This may be due to the differences between the expectations of each participant. As experiences lead to certain expectations and expectations differ as we get older, perception of people from different age groups can be different.

The evaluation of the relation between the gender of the participants and the ligbting șistems

Chi-square distribution is 6.32072 that is smaller than the computed $c^{\prime}(d f=4, a=0.050)=9.49$, therefore the null h!̣othesis is accepted at 5 percent level of significance. The perception of a space under different lighting arrangements is independent of the sex of the participants.

The evaluation of the relation beturen the eye deficiencies of the participants and the lighting spstems

The results of the participants who do not have any eye deficiencies were compared with the ones who have. Chi-square distribution is 5.977088 that is smaller than compured $c^{2}(d f=i, a=0.050)=9.49$. therefore the null hypothesis is accepted at 5 percent level of significance, there is no relation between the eye defects of the participants and the perception of a space under different lighting arrangements. We see through our eves, but the process of perception ends in the brain. Most of the participants who had eve defects were using eve glasses. $s$ ) the deficiencies were corrected by suitable lenses and they perceired as the ones who do not have anv eve defects.

\section{Discussion and Conclusions}

The results show that different lighting arrangements influence perception. Under different lighting arrangements, participants used different words to define the same space. This result supports Flynn's findings [3] When the preferred lighting system for each impression is considered. 
Wall washing enhanced the impressions of clarity and order. TL54 fluorescent rubes which are cooler light sources and provide uniform illumination were selected for this lighting system. These properties of the light sources can be effective for clarity and order, or this result can be related to the illumination levels. When the illumination levels were measured, out of the four lighting systems, the highestvalue was achieved under wall washing as can be seen in Table 2. This result support's the findings of Hughes and his associates [10] .

Cove lighting was the lighting system that increased the impressions of spaciousness and order. II54 fluorescent tubes were selected for this lighting system as well. These are cooler light sources and are preferred to warmer toned light sources (tungsten-halogenated lamps) and this is parallel to the findings of Aksugür [11] .

Uplighting was preferred for the impressions of pleasantness, privacy and relaxation. Colour of the light source and the distribution characteristics are more important than the illumination levels for these impressions as Table 2 shows. Though existing fluorescent tubes and tungsten-halogenated lamps provided same illumination levels, there was a large difference between the response rates. This result is in accordance with Baron and Rea [8] who concluded that people reported more positive feelings under warm light.

\section{Table 2}

Illumination Levels for Each Lighting System

\begin{tabular}{|lc|}
\hline & illumination levels (lx) \\
\hline existing fluorescent tubes & 110 \\
cove lighting & 270 \\
wall washing & 300 \\
uplighting & 110 \\
\hline
\end{tabular}

According to the results of the study, it has been found out that age is an important component for the process of perception, however eye deficiencies and the gender of the participants did not affect perception.

\section{Acknowledgements}

We would like to thank Dursun Manav for his assistance with data analysis stage and the Institute of Fine Ars of Bilkent University for financial suppor.

\section{References}

1. P.C. SORCAR:Architectural ligbting Design for Commercial lnteriors. John Wiiey and Sons, New York, 1987. pp.272.

2. L. ERHARDT: Views on the Visual Environment. Illuminating Engineering Sociery of North America, New York, 1985. pp.50.

3. J.E. FLYNN et al: Interim Study of Procedures for Investigating the Effect of Light on Impression and Behavior. Selected Papers on Arcbitectural Ligbting. Ed. Mark Rea. SPIE Optical Engineering Press, Washington, 1992.

4. C. HENDRICKetal: Procedures for Investigating the Effect of Light on Impressions - Simulation of a Real Space by Slides. Environment and Bebavior. Vol 9 (1977), pp.491-510.

5 J.E. FLYNN: Lighting Design Decisions As Interventions in Human Space. Environmental Aesthetics- Theory, Research and Application. Ed. Jack Nasar. Cambridge University Press, New York, 1992.

6. D. L. BUTLER and P. M. BINER: Preferred Light Levels-Variability Among Settings, Behaviors and Individuals. Environment and Bebavior, Vol. 19 (1987), pp.695-721.

7. F. K. SMITH: Spaciousness. Lighting Design and Application, Sep (1989), pp.18-23.

8. R. A. BARON and M. S. REA. Lighting to Soothe the Mood. Lighting Design and Application, Dec (1991), pp.30-32.

9. D.LOE: The Power of Lighting. Lighting Design and Application. Sep (1989), pp.18-23.

10. P. C. HUGHES et al: An Examination of Visual Clarity As a Function of Colour Temperature of Light Sources. Lighting Design and Application, July (1977), pp.22.

11. E. AKSUGÜR: Effects of Surface Colours of Walls Under Different Light Sources on the Perceptual Magnitude of a Space in a Room. Colour 77: Proceedings of the Third Congress of the International Color Association, New York, 10-15 July 1977, Ed. Fred W Billmeyer Jr and Gunter Wyszecki. Adam Hilger, Bristol, 1977, pp.388-391. 